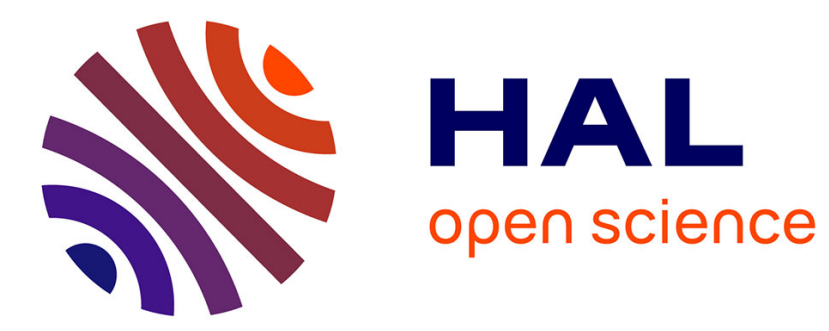

\title{
Lack of knowledge in structural model validation
}

Pierre Ladevèze, Guillaume Puel, Thierry Romeuf

\section{To cite this version:}

Pierre Ladevèze, Guillaume Puel, Thierry Romeuf. Lack of knowledge in structural model validation. Computer Methods in Applied Mechanics and Engineering, 2006, 195 (37-40), pp.4697-4710. 10.1016/j.cma.2005.10.017 . hal-00258641

\section{HAL Id: hal-00258641 \\ https://hal.science/hal-00258641}

Submitted on 22 Feb 2008

HAL is a multi-disciplinary open access archive for the deposit and dissemination of scientific research documents, whether they are published or not. The documents may come from teaching and research institutions in France or abroad, or from public or private research centers.
L'archive ouverte pluridisciplinaire HAL, est destinée au dépôt et à la diffusion de documents scientifiques de niveau recherche, publiés ou non, émanant des établissements d'enseignement et de recherche français ou étrangers, des laboratoires publics ou privés. 


\title{
Lack Of Knowledge in Structural Model Validation
}

\author{
P. Ladevèze ${ }^{a, *}$, G. Puel $^{\mathrm{a}, 1}$, T. Romeuf ${ }^{\mathrm{b}}$ \\ ${ }^{\text {a } L M T-C a c h a n ~(E N S ~ C a c h a n / C N R S / P a r i s ~} 6$ University) \\ 61 avenue du Président Wilson, 94235 Cachan Cedex, France \\ ${ }^{\mathrm{b}}$ European Aeronautic Defence and Space Company (EADS Space Transportation), \\ Route de Verneuil, BP 96, 78133 Les Mureaux Cedex, France
}

\begin{abstract}
This paper deals with the structural modeling of a family of similar, actual structures taking into account uncertainties and modeling errors. Only errors of the "structural stiffness" type are considered. We develop a new theory in which what we call the Lack Of Knowledge (LOK) is defined through an internal variable, whose upper and lower bounds are stochastic, associated with each substructure. Two main questions are discussed: the impact of the basic LOKs on the predicted structural response and the reduction of the basic LOKs through the use of additional information.
\end{abstract}

Key words: modeling, validation, lack of knowledge, structure, dynamics

\section{Introduction}

Today, the validation of complex structural models - i.e. the assessment of their quality - remains a major issue. The objective is to quantify, then to improve, the quality of the structural model being used by comparison with an experimental reference. Most advanced approaches rely on the updating of deterministic dynamic parameters (stiffness, mass, damping) based on free or forced vibration tests $[1,2]$. Probabilistic models are investigated in [3-5]. The

\footnotetext{
* Corresponding author

Email addresses: pierre.ladeveze@lmt.ens-cachan.fr (P. Ladevèze), guillaume.puel@ecp.fr (G. Puel), thierry.romeuf@space.eads.net

(T. Romeuf).

1 Present address: LMSSMat (École Centrale Paris/CNRS)

Grande Voie des Vignes, 92295 Châtenay Malabry Cedex, France
} 
final result is an updated structural model which, evidently, cannot reproduce the behavior of the family of actual structures under consideration perfectly.

In order to describe some of the uncertainties as material uncertainties, the use of probabilistic methods has become increasingly popular [6]. These methods consist in studying the effect of the uncertainties affecting the input on the variability of the output, which can be achieved in various ways and has led to major improvements. For example, many stochastic finite element methods have been proposed [7-11]. There are also methods which do not necessarily involve probability laws and which are capable of dealing with uncertainties unrelated to the actual variability of a given parameter [12-14].

Here, we follow quite a different approach, and the structural model being used can be deterministic or not. Evidently, such a model does not yield a "perfect" prediction of the response. There are numerous sources of uncertainties as well as modeling errors, which we describe using the concept of Lack Of Knowledge [15-18]. The structure being studied is defined as an assembly of substructures $E$ in which the connections can be viewed as special substructures. The LOKs are defined on the substructure level: the basic LOK $m_{\mathrm{E}}$ is a scalar internal variable which quantifies the substructure's LOK state. In mathematical terms, $m_{\mathrm{E}}$ is bounded by $m_{\mathrm{E}}^{+}(\theta)$ and $m_{\mathrm{E}}^{-}(\theta)$, which follow probabilistic laws.

In this paper, we limit ourselves to Lack Of Knowledge of the "structural stiffness" type and to structures described as deterministic FE dynamic models. The LOK theory is developed with particular emphasis on two aspects:

- the meaning of what we call a structural model with LOKs, and its impact on the prediction of the structural response,

- the reduction of the basic LOKs using additional information.

Finally, we conclude by giving some engineering illustrations.

\section{Basic LOKs}

To define the framework of our theory, let us consider a family of actual, quasiidentical structures, each being modeled as an assembly of substructures $E$ in which the connections can be viewed as special substructures. We assume that a deterministic FE model has been defined in order to predict the response of the "structure". All quantities associated with the FE model are designated by $\boldsymbol{\bullet}$. Only errors of the "structural stiffness" type are considered. 


\subsection{Definition of the basic LOKs}

We associate with each substructure $E$ of an actual structure $\Omega$ a LOK variable $m_{\mathrm{E}}$ defined over an interval whose bounds $m_{\mathrm{E}}^{+}(\theta)$ and $m_{\mathrm{E}}^{-}(\theta)$ are formally defined as follows:

$$
\left(1-m_{\mathrm{E}}^{-}(\theta)\right) \overline{\mathbf{K}}_{\mathrm{E}} \leq \mathbf{K}_{\mathrm{E}} \leq\left(1+m_{\mathrm{E}}^{+}(\theta)\right) \overline{\mathbf{K}}_{\mathrm{E}}
$$

$\overline{\mathbf{K}}_{\mathrm{E}}$ designates the stiffness matrix of the FE model being used. $\mathbf{K}_{\mathrm{E}}$ is the stiffness matrix of an actual structure belonging to the family under consideration. In this formal expression, the inequalities can be considered to hold for the eigenvalues associated with these matrices.

In practice, these inequalities are expressed using the strain energies:

$$
\left(1-m_{\mathrm{E}}^{-}(\theta)\right) \bar{e}_{\mathrm{E}} \leq e_{\mathrm{E}} \leq\left(1+m_{\mathrm{E}}^{+}(\theta)\right) \bar{e}_{\mathrm{E}}
$$

where $e_{\mathrm{E}}=\frac{1}{2} U^{T} \mathbf{K}_{\mathrm{E}} U$ denotes the strain energy of an actual structure belonging to the family of similar structures being studied, and $\bar{e}_{\mathrm{E}}=\frac{1}{2} U^{T} \overline{\mathbf{K}}_{\mathrm{E}} U$ the strain energy of the theoretical model. These inequalities must hold for any displacement field $U$. The two quantities $m_{\mathrm{E}}^{+}(\theta)$ and $m_{\mathrm{E}}^{-}(\theta)$ are scalar internal variables of Substructure $E$, which we call the upper basic $L O K$ and the lower basic $L O K$ respectively. These are stochastic variables.

For each substructure, the LOK $m_{\mathrm{E}}$ lies within the interval $\left[-m_{\mathrm{E}}^{-} ; m_{\mathrm{E}}^{+}\right]$, and one cannot be more precise than that. As we will see later, this description is compatible with a rigorous analysis of the LOKs on the response of the structure. Let us consider two particular examples of LOK definitions:

- for a uniform probability law, $m_{\mathrm{E}}$ belongs to intervals $\left[-m_{\mathrm{E}}^{-} ; m_{\mathrm{E}}^{+}\right]$, and the range of the uniform probability law associated to these bounds is an interval denoted $\left[-\bar{m}_{\mathrm{E}}^{-} ; \bar{m}_{\mathrm{E}}^{+}\right]$;

- other cases can be described by an interval $\left[-\bar{m}_{\mathrm{E}}^{-} ; \bar{m}_{\mathrm{E}}^{+}\right]$without giving any probabilistic law. One reverts to the simple interval analysis mentioned in $[19,20]$, which can be used, for example, to describe the LOK related to an actual nonlinear connection calculated using a linear model.

Other situations can be envisaged, such as the use of a normal probability law, which is detailed in the following section.

\subsection{Illustration: material variability}

When the only sources of errors being considered are those related to the material, one can reasonably assume that the LOK on Sub- 
structure $E$ is a stochastic variable $m_{\mathrm{E}}(\theta)$ following a modified normal law $p^{*}\left(m_{\mathrm{E}}\right)$ based on the classical normal probability density $p\left(m_{\mathrm{E}}\right)=\frac{1}{\sqrt{2 \pi \sigma^{2}}} e^{-\frac{m_{\mathrm{E}}^{2}}{2 \sigma^{2}}}$ :

$$
p^{*}\left(m_{\mathrm{E}}\right)=\left\{\begin{array}{cl}
A \cdot p\left(m_{\mathrm{E}}\right) & \text { if } m_{\mathrm{E}} \in\left[-\bar{m}_{\mathrm{E}}^{-} ; \bar{m}_{\mathrm{E}}^{+}\right] \\
0 & \text { if } \left.m_{\mathrm{E}} \in\right]-\infty ;-\bar{m}_{\mathrm{E}}^{-}[\cup] \bar{m}_{\mathrm{E}}^{+} ; \infty[
\end{array}\right.
$$

This law can be considered as a truncated normal law, where a zero probability density outside $\left[-\bar{m}_{\mathrm{E}}^{-} ; \bar{m}_{\mathrm{E}}^{+}\right]$is prescribed in order to eliminate nonphysical samples $\left(-m_{\mathrm{E}}^{-}<-1\right)$. The quantity $\sigma$ coming from the normal law can be defined using the quantities $\bar{m}_{\mathrm{E}}^{+}$and $\bar{m}_{\mathrm{E}}^{-}$, for example by setting $\int_{-\bar{m}_{\mathrm{E}}^{-}}^{\bar{m}_{\mathrm{E}}^{+}} p\left(m_{\mathrm{E}}\right) \mathrm{d} m_{\mathrm{E}}=0.99$. In this case, the normalization constant $A$ equals to $\frac{1}{0.99}$.

We want now to describe this stochastic variable in terms of Lack of Knowledge. With such a law, the probability of observing $m_{\mathrm{E}}(\theta)$ in a given interval $\left[-m_{\mathrm{E}}^{-} ; m_{\mathrm{E}}^{+}\right]$is:

$$
P\left(-m_{\mathrm{E}}^{-} \leq m_{\mathrm{E}}(\theta) \leq m_{\mathrm{E}}^{+}\right)=\int_{-m_{\mathrm{E}}^{-}}^{m_{\mathrm{E}}^{+}} p\left(m_{\mathrm{E}}\right) \mathrm{d} m_{\mathrm{E}}
$$

Since in (2) the basic LOKs $m_{\mathrm{E}}^{+}(\theta)$ and $m_{\mathrm{E}}^{-}(\theta)$ are defined on both sides of the theoretical model, the previous situation can be described by two mutually exclusive events:

- either $m_{\mathrm{E}} \in\left[0 ; m_{\mathrm{E}}^{+}\right]$, i.e. one has the event $\left(m_{\mathrm{E}}^{-}=0, m_{\mathrm{E}}^{+} \geq 0\right)$ with the probability $P^{+}\left(m_{\mathrm{E}}^{+}\right)$;

- or $m_{\mathrm{E}} \in\left[-m_{\mathrm{E}}^{-} ; 0\right]$, i.e. one has the event $\left(m_{\mathrm{E}}^{-} \geq 0, m_{\mathrm{E}}^{+}=0\right)$ with the probability $P^{-}\left(m_{\mathrm{E}}^{-}\right)$.

This statement describes how we compute the basic LOKs in practice: depending on the value of $m_{\mathrm{E}}$ obtained by random drawing, one has two distinct types of intervals, $\left[0 ; m_{\mathrm{E}}^{+}\right]$and $\left[-m_{\mathrm{E}}^{-} ; 0\right]$, whose collection constitutes the basic LOK on Substructure $E$. This case illustrates how one can represent an uncertainty defined by a classical probability law using our concept of Lack Of Knowledge.

\subsection{Interval probability}

Since, in practice, the use of two probabilities $P^{+}$and $P^{-}$is relatively complex, we decided in [21] to introduce a number of mathematical tools; of course, other ways could have been considered. 
Let us consider a family of intervals $\left[-m_{\mathrm{E}}^{-} ; m_{\mathrm{E}}^{+}\right] \ni m_{\mathrm{E}}$ with $m_{\mathrm{E}}^{+}+m_{\mathrm{E}}^{-}=L$. The interval $\left[-m_{\mathrm{E}}^{-} ; m_{\mathrm{E}}^{+}\right]$is said to be standard if for a given $L$, the probability of having $m_{\mathrm{E}}$ in $\left[-m_{\mathrm{E}}^{-} ; m_{\mathrm{E}}^{+}\right]$is maximum over the set of all intervals with length $L$ :

$$
I(L)=\arg \max _{\substack{\left[-m_{\mathrm{E}}^{-} ; m_{\mathrm{E}}^{+}\right] \\ m_{\mathrm{E}}^{+}+m_{\mathrm{E}}^{-}=L}} P^{+}\left(m_{\mathrm{E}}^{+}\right)+P^{-}\left(m_{\mathrm{E}}^{-}\right)
$$

With this definition, one can introduce the concept of interval probability by stating that for a given $L P(L)$ is the probability associated with the definition (5) of $I(L)$ :

$$
P(L)=\max _{\substack{\left[-m_{\mathrm{E}}^{-} ; m_{\mathrm{E}}^{+}\right] \\ m_{\mathrm{E}}^{+}+m_{\mathrm{E}}^{-}=L}} P^{+}\left(m_{\mathrm{E}}^{+}\right)+P^{-}\left(m_{\mathrm{E}}^{-}\right)
$$

Thus, $P^{+}\left(m_{\mathrm{E}}^{+}\right)$and $P^{-}\left(m_{\mathrm{E}}^{-}\right)$being linked to the distribution functions of the occurrences of the upper and lower bounds $m_{\mathrm{E}}^{+}$and $m_{\mathrm{E}}^{-}$respectively, the probability of the uncertain variable $m_{\mathrm{E}}$ being within $\left[-m_{\mathrm{E}}^{-} ; m_{\mathrm{E}}^{+}\right]$is greater than $P^{+}\left(m_{\mathrm{E}}^{+}\right)+P^{-}\left(m_{\mathrm{E}}^{-}\right)$: indeed, $P^{+}\left(m_{\mathrm{E}}^{+}\right)+P^{-}\left(m_{\mathrm{E}}^{-}\right)<1$ means that by sampling larger intervals containing $\left[-m_{\mathrm{E}}^{-} ; m_{\mathrm{E}}^{+}\right]$the probability of $m_{\mathrm{E}}$ being within these intervals would be higher.

Another possible interpretation of these definitions is that if one seeks an interval of basic LOKs such that $m_{\mathrm{E}}$ has at least a given probability $P$ of being inside, one must consider the standard interval $I\left(L_{\mathrm{P}}\right)$ such that the associated interval probability $P\left(L_{\mathrm{P}}\right)$ equals $P$. One can show that this interval $I\left(L_{\mathrm{P}}\right)$ is, in fact, the smallest interval $\left[-m_{\mathrm{E}}^{-} ; m_{\mathrm{E}}^{+}\right]$such that $P^{+}\left(m_{\mathrm{E}}^{+}\right)+P^{-}\left(m_{\mathrm{E}}^{-}\right)=P$, which can be expressed mathematically as:

$$
I\left(L_{\mathrm{P}}\right)=\arg \min _{\substack{\left[-m_{\mathrm{E}}^{-} ; m_{\mathrm{E}}^{+}\right] \\ P^{+}\left(m_{\mathrm{E}}^{+}\right)+P^{-}\left(m_{\mathrm{E}}^{-}\right)=P}} m_{\mathrm{E}}^{+}+m_{\mathrm{E}}^{-}
$$

In the case of uniform laws, uniqueness is not guaranteed, but the important point is to be able to consider a family of standard intervals defined by only one stochastic variable, which simplifies the analysis.

\section{Structural modeling with LOKs}

\subsection{The new structural model}

Let us recall that we are considering only LOKs of the stiffness type. The basic LOKs are assumed to be known, and so is the FE operator $\overline{\mathbb{A}}$ used to 
calculate the response $\bar{s}$ of the structure over the time-space domain:

$$
\bar{s}=\overline{\mathbb{A}}\left(\overline{\mathbf{K}}_{\mathrm{E}} ; E \in \Omega\right)
$$

$\left\{\overline{\mathbf{K}}_{\mathrm{E}}, E \in \Omega\right\}$ should be viewed as parameters. One assumes that Relation (8) holds for any actual structure belonging to the family under consideration if the stiffness matrices are replaced by the actual stiffnesses:

$$
s=\overline{\mathbb{A}}\left(\mathbf{K}_{\mathrm{E}} ; E \in \Omega\right)
$$

The relation between the actual stiffness and the FE stiffness is established through the basic LOKs:

$$
\left(1-m_{\mathrm{E}}^{-}(\theta)\right) \overline{\mathbf{K}}_{\mathrm{E}} \leq \mathbf{K}_{\mathrm{E}} \leq\left(1+m_{\mathrm{E}}^{+}(\theta)\right) \overline{\mathbf{K}}_{\mathrm{E}} \quad \forall E \in \Omega
$$

Indeed, in a certain way, we are defining an envelope of the actual responses. Let us consider a scalar quantity of interest $\alpha$ :

$$
\alpha=\widehat{\alpha}(s)
$$

where $\widehat{\alpha}$ is a given operator. Let us define:

$$
\Delta \alpha_{\mathrm{mod}}=\alpha-\bar{\alpha}
$$

where $\bar{\alpha}$ is the $\mathrm{FE}$ value, ie:

$$
\bar{\alpha}=\widehat{\alpha}(\bar{s})
$$

For the quantity of interest $\alpha$, one defines the following envelope of the possible actual responses:

$$
-\left[\Delta \alpha_{\text {mod }}^{-}\right](\theta) \leq\left[\Delta \alpha_{\text {mod }}\right] \leq\left[\Delta \alpha_{\text {mod }}^{+}\right](\theta)
$$

These can be obtained from Problems (15) and (18). Let us start with $\left[\Delta \alpha_{\text {mod }}^{+}\right](\theta)$, which is determined from the problem:

$$
\Delta \alpha_{\text {mod }}^{+}=\max _{-m_{\mathrm{E}}^{-} \overline{\mathbf{K}}_{\mathrm{E}} \leq \mathbf{K}_{\mathrm{E}}-\overline{\mathbf{K}}_{\mathrm{E}} \leq m_{\mathrm{E}}^{+} \overline{\mathbf{K}}_{\mathrm{E}}} \widehat{\alpha}\left(\overline{\mathbb{A}}\left(\mathbf{K}_{\mathrm{E}} ; E \in \Omega\right)\right)-\bar{\alpha}
$$

Consequently, $\Delta \alpha_{\bmod }^{+}$can be expressed as a given function of the quantities $\left(m_{\mathrm{E}}^{+} ; m_{\mathrm{E}}^{-}\right)_{E \in \Omega}$ :

$$
\Delta \alpha_{\text {mod }}^{+}=\mathbb{Z}^{+}\left(m_{\mathrm{E}}^{+}, m_{\mathrm{E}}^{-} ; E \in \Omega\right)
$$

and remembering that these quantities are stochastic variables, we can finally write:

$$
\left[\Delta \alpha_{\text {mod }}^{+}\right](\theta)=\mathbb{Z}^{+}\left(m_{\mathrm{E}}^{+}(\theta), m_{\mathrm{E}}^{-}(\theta) ; E \in \Omega\right)
$$


For $\Delta \alpha_{\text {mod }}^{-}(\theta)$, one first solves:

$$
-\Delta \alpha_{\text {mod }}^{-}=\min _{-m_{\mathrm{E}}^{-} \overline{\mathbf{K}}_{\mathrm{E}} \leq \mathbf{K}_{\mathrm{E}}-\overline{\mathbf{K}}_{\mathrm{E}} \leq m_{\mathrm{E}}^{+} \overline{\mathbf{K}}_{\mathrm{E}}} \widehat{\alpha}\left(\overline{\mathbb{A}}\left(\mathbf{K}_{\mathrm{E}} ; E \in \Omega\right)\right)-\bar{\alpha}
$$

then:

$$
\begin{gathered}
\Delta \alpha_{\text {mod }}^{-}=\mathbb{Z}^{-}\left(m_{\mathrm{E}}^{+}, m_{\mathrm{E}}^{-} ; E \in \Omega\right) \\
{\left[\Delta \alpha_{\text {mod }}^{-}\right](\theta)=\mathbb{Z}^{-}\left(m_{\mathrm{E}}^{+}(\theta), m_{\mathrm{E}}^{-}(\theta) ; E \in \Omega\right)}
\end{gathered}
$$

Remarks:

- The resolution of Problems (15) and (18) is not very difficult, especially if the basic LOKs are small, in which case linearization procedures can be used, like in Section 3.2. A more general case is studied in Section 3.3.

- What we now call the structural model consists of both the operator $\overline{\mathbb{A}}$ and the basic LOKs.

Let us proceed a little further and introduce an interval probability $P_{\alpha}(L)$ such that:

$$
P\left(\Delta \alpha_{\text {mod }} \in I_{\Delta \alpha_{\text {mod }}}(L)\right)=P_{\alpha}(L) \forall L
$$

Again, this is the same as considering, for a given probability $P$, the standard interval $I_{\Delta \alpha_{\text {mod }}}\left(L_{\mathrm{P}}\right)$ such that $P\left(\Delta \alpha_{\text {mod }} \in I_{\Delta \alpha_{\text {mod }}}\left(L_{\mathrm{P}}\right)\right)$ is greater than $P$. The two bounds of this interval $I_{\Delta \alpha_{\text {mod }}}\left(L_{\mathrm{P}}\right)$, denoted $\Delta \alpha_{\text {mod }}^{-}(P)$ and $\Delta \alpha_{\text {mod }}^{+}(P)$, constitute what we call the effective $L O K$ on the quantity of interest $\alpha$. Thus, whereas the initial theoretical model gives us an incomplete description of the actual phenomena, the addition of a LOK-model easily makes possible to derive more detailed information about the quantity of interest $\alpha$ through the calculation of confidence intervals. The question of the determination of this LOK-model will be addressed in Section 4.

\subsection{Effective LOKs for dynamic problems}

The calculation of effective LOKs can be applied to quantities of interest which are common in modal analysis. Here, we are interested in free vibrations; therefore, we are using as the quantities of interest $\alpha$ the eigenfrequencies $\omega_{i}$ (in $\mathrm{rd} / \mathrm{s}$ ) and eigenmodes $\underline{\phi}_{i}$ which are defined by the eigenvalue problem:

$$
\left(\mathbf{K}-\omega_{i}^{2} \mathbf{M}\right) \underline{\phi}_{i}=\underline{0}
$$


where $\mathbf{K}$ and $\mathbf{M}$ are the global stiffness and mass matrices respectively. Moreover, the global stiffness matrix can be split among the different substructures $E$ of the structure $\Omega: \mathbf{K}=\sum_{E \in \Omega} \mathbf{K}_{\mathrm{E}}$.

Let us recall that the pair $\Delta \alpha_{\text {mod }}^{-}(P), \Delta \alpha_{\text {mod }}^{+}(P)$ constitutes what we call the effective $L O K$ related to the quantity $\Delta \alpha_{\text {mod }}$. The determination of these two quantities for eigenfrequencies and eigenmodes, described in the following two sections, relies on the rigorous propagation of the basic LOK intervals using relations between the eigenmodes of an original model and those of a perturbed model, a classical practice in modal analysis.

Writing the difference of the eigenvalue problems (22) for both models, the system to be solved is, with a first-order approximation:

$$
\overline{\mathbf{Z}}\left(\bar{\omega}_{i}\right) \Delta \underline{\phi}_{i \bmod }=\underline{B}\left(\bar{\omega}_{i}\right)
$$

where:

$$
\begin{aligned}
\overline{\mathbf{Z}}\left(\bar{\omega}_{i}\right) & =\left(\overline{\mathbf{K}}-\bar{\omega}_{i}^{2} \overline{\mathbf{M}}\right) \\
\underline{B}\left(\bar{\omega}_{i}\right) & =-\left(\Delta \mathbf{K}-\Delta \omega_{i \bmod }^{2} \overline{\mathbf{M}}\right) \bar{\phi}_{i}
\end{aligned}
$$

and the two models are linked by:

$$
\begin{aligned}
\mathbf{K} & =\overline{\mathbf{K}}+\Delta \mathbf{K} \\
\mathbf{M} & =\overline{\mathbf{M}} \\
\omega_{i \mathrm{mod}}^{2} & =\bar{\omega}_{i}^{2}+\Delta \omega_{i \mathrm{mod}}^{2} \\
\underline{\phi}_{i \mathrm{mod}} & =\bar{\phi}_{i}+\Delta \underline{\phi}_{i \mathrm{mod}}
\end{aligned}
$$

Since the matrix $\bar{Z}$ is singular at $\bar{\omega}_{i}$, there are solutions of (23) if and only if:

that is:

$$
\underline{\phi}_{i}^{T} \underline{B}\left(\bar{\omega}_{i}\right)=0
$$

$$
\underline{\bar{\phi}}_{i}^{T} \mathbf{M} \bar{\phi}_{i} \Delta \omega_{i \text { mod }}^{2}=\underline{\phi}_{i}^{T} \Delta \mathbf{K} \bar{\phi}_{i}
$$

In this case, solutions could be expressed as:

$$
\Delta \underline{\phi}_{i \bmod }=\underline{\psi}_{i}+a_{i} \underline{\phi}_{i}
$$

where $\psi_{i}$ is a particular solution, one given coordinate of which is set to zero [22].

\subsubsection{Effective LOK on an eigenfrequency}

If the modes $\bar{\phi}_{i}$ of the theoretical model are normalized with respect to the mass matrix $\left(\underline{\phi}_{j}^{T} \overline{\mathbf{M}}_{i}=\delta_{j i}\right)$, one can express the discrepancy 
between $\omega_{i}^{2}$ and $\bar{\omega}_{i}^{2}$ according to $(28)$ as:

$$
\Delta \omega_{i \text { mod }}^{2}=\underline{\phi}_{i}^{T}(\mathbf{K}-\overline{\mathbf{K}}) \bar{\phi}_{i}=2 \sum_{E \in \Omega}\left(e_{\mathrm{E}}\left(\bar{\phi}_{i}\right)-\bar{e}_{\mathrm{E}}\left(\bar{\phi}_{i}\right)\right)
$$

by definition of the strain energies, and when the basic LOKs are small enough to use the condition (28). When there are multiple modes, other expressions can be used $[23,24]$.

With the previous equation, the fundamental relation (2) allows a rigorous propagation of the intervals $\left(\left[-m_{\mathrm{E}}^{-} ; m_{\mathrm{E}}^{+}\right]\right)_{E \in \Omega}$ (where, for each substructure $E$, $\left(m_{\mathrm{E}}^{-}, m_{\mathrm{E}}^{+}\right)$is a particular sample of the basic LOKs depending on the probability laws chosen) as follows:

$$
-\left[\Delta \omega_{i \mathrm{mod}}^{2-}\right](\theta) \leq\left[\Delta \omega_{i \mathrm{mod}}^{2}\right] \leq\left[\Delta \omega_{i \mathrm{mod}}^{2+}\right](\theta)
$$

with

$$
\begin{aligned}
& {\left[\Delta \omega_{i \text { mod }}^{2-}\right](\theta)=\mathbb{Z}^{-}\left(m_{\mathrm{E}}^{+}(\theta), m_{\mathrm{E}}^{-}(\theta) ; E \in \Omega\right)=2 \sum_{E \in \Omega} m_{\mathrm{E}}^{-}(\theta) \bar{e}_{\mathrm{E}}\left(\underline{\phi}_{i}\right)} \\
& {\left[\Delta \omega_{i \text { mod }}^{2+}\right](\theta)=\mathbb{Z}^{+}\left(m_{\mathrm{E}}^{+}(\theta), m_{\mathrm{E}}^{-}(\theta) ; E \in \Omega\right)=2 \sum_{E \in \Omega} m_{\mathrm{E}}^{+}(\theta) \bar{e}_{\mathrm{E}}\left(\bar{\phi}_{i}\right)}
\end{aligned}
$$

If the probability laws for the basic LOKs are known, one can obtain the dispersions of the bounds $\Delta \omega_{i \bmod }^{2-}$ and $\Delta \omega_{i \bmod }^{2+}$ and, thus, determine for a given probability $P$ the two bounds $\left[\Delta \omega_{i \bmod }^{2-}\right](\theta)$ and $\left[\Delta \omega_{i \text { mod }}^{2+}\right](\theta)$ of the associated standard interval $I_{\Delta \omega_{i \text { mod }}^{2}}\left(L_{\mathrm{P}}\right)$. In other words, one obtains the effective LOK on the square of the eigenfrequency $\omega_{i}^{2}$.

\subsubsection{Effective $L O K$ on an eigenmode}

Since a mode $\underline{\phi}_{i}$ is not a scalar quantity, one is interested either in its component $\underline{\phi}_{k i}$ along a specific degree of freedom $k$ or in its projection $\phi_{i}^{\mathrm{N}}$ onto a given direction $\underline{N}$. In either case, for small LOKs $\left(m_{\mathrm{E}}^{-}(\theta), m_{\mathrm{E}}^{+}(\theta)\right)_{E \in \Omega}$, one can express the dispersions of the bounds $\left[\Delta \phi_{k i \text { mod }}^{-}\right](\theta)$ and $\left[\Delta \phi_{k i \text { mod }}^{+}\right](\theta)$ as well as $\left[\Delta \phi_{i \mathrm{mod}}^{\mathrm{N}-}\right](\theta)$ and $\left[\Delta \phi_{i \mathrm{mod}}^{\mathrm{N}+}\right](\theta)$ as linear combinations of the basic LOKs: the resulting relations come from Nelson's classical formula (29). Let us remark that in the case of industrial structures with a great number of DOFs, reduction bases can be used and associated perturbation expressions are derived [25].

For example, for the calculation of the bounds $\left[\Delta \phi_{i \text { mod }}^{\mathrm{N}-}\right](\theta)$ and $\left[\Delta \phi_{i \bmod }^{\mathrm{N}+}\right](\theta)$, one obtains the following relations:

$$
-\left[\Delta \phi_{i \mathrm{mod}}^{\mathrm{N}-}\right](\theta) \leq\left[\left(\underline{\phi}_{i}-\underline{\phi}_{i}\right) \cdot \underline{N}\right] \leq\left[\Delta \phi_{i \mathrm{mod}}^{\mathrm{N}+}\right](\theta)
$$


with

$$
\begin{aligned}
{\left[\Delta \phi_{i \mathrm{mod}}^{\mathrm{N}-}\right](\theta) } & =\mathbb{Z}^{-}\left(m_{\mathrm{E}}^{+}(\theta), m_{\mathrm{E}}^{-}(\theta) ; E \in \Omega\right) \\
& =\sum_{E \in \Omega} \sum_{\substack{k=1 \\
k \neq i}}^{r} \frac{1}{2\left(\bar{\omega}_{k}^{2}-\bar{\omega}_{i}^{2}\right)}\left\{m_{\mathrm{E}}^{-}(\theta) \bar{e}_{\mathrm{E}}\left(\underline{\phi}_{i}+\underline{\phi}_{k}\right)+m_{\mathrm{E}}^{+}(\theta) \bar{e}_{\mathrm{E}}\left(\bar{\phi}_{i}-\bar{\phi}_{k}\right)\right\} \bar{\phi}_{k} \cdot \underline{N} \\
{\left[\Delta \phi_{i \mathrm{mod}}^{\mathrm{N}+}\right](\theta) } & =\mathbb{Z}^{+}\left(m_{\mathrm{E}}^{+}(\theta), m_{\mathrm{E}}^{-}(\theta) ; E \in \Omega\right) \\
& =\sum_{E \in \Omega} \sum_{\substack{k=1 \\
k \neq i}}^{r} \frac{1}{2\left(\bar{\omega}_{k}^{2}-\bar{\omega}_{i}^{2}\right)}\left\{m_{\mathrm{E}}^{+}(\theta) \bar{e}_{\mathrm{E}}\left(\underline{\phi}_{i}+\underline{\phi}_{k}\right)+m_{\mathrm{E}}^{-}(\theta) \bar{e}_{\mathrm{E}}\left(\underline{\phi}_{i}-\underline{\phi}_{k}\right)\right\} \underline{\phi}_{k} \cdot \underline{N}
\end{aligned}
$$

Then, for a given probability $P$, one determines the two bounds $\Delta \phi_{i \text { mod }}^{\mathrm{N}-}(P)$ and $\Delta \phi_{i \text { mod }}^{\mathrm{N}+}(P)$ of the associated standard interval $I_{\Delta \phi_{i \text { mod }}^{\mathrm{N}}}\left(L_{\mathrm{P}}\right)$. In other words, one obtains the effective LOK on the value of the projection $\phi_{i} \cdot \underline{N}$. More details can be found in [15].

\subsection{Calculation of effective LOKs when a given basic LOK is large}

All of the above derivations of effective LOKs are based on the expressions of the variations of modal quantities in a first-order approximation: this is correct provided the basic LOKs on the substructures are all small enough. The objective of this section is to define a new way of calculating the effective LOKs in the presence of a large basic LOK.

\subsubsection{Principle}

Let us assume that large values of the basic LOK $m_{\mathcal{E}}(\theta)$ defined on Substructure $\mathcal{E}$ can be sampled. The idea is to calculate the eigenfrequencies $\widetilde{\omega}_{i}\left(m_{\mathcal{E}}\right)$ and eigenmodes $\widetilde{\phi}_{i}\left(m_{\mathcal{E}}\right)$ of the structure perturbed by the large value of the given sample $m_{\mathcal{E}}$ : let us recall that this value is either $m_{\mathcal{E}}^{+}$, or $-m_{\mathcal{E}}^{-}$, depending on the type of interval drawn (either $\left[0 ; m_{\mathcal{E}}^{+}\right]$, or $\left[-m_{\mathcal{E}}^{-} ; 0\right]$ ).

The actual problem to be solved is to find $\widetilde{\omega}_{i}$ and $\widetilde{\phi}_{i}$ such that:

$$
\left(\overline{\mathbf{K}}+m_{\mathcal{E}} \Delta \mathbf{K}_{\mathcal{E}}\right) \tilde{\phi}_{i}=\widetilde{\omega}_{i}^{2} \mathbf{M} \underline{\phi}_{i}
$$

where $\Delta \mathbf{K}_{\mathcal{E}}$ is the contribution of Substructure $\mathcal{E}$ to the stiffness matrix of the theoretical model. If one seeks to keep the computational cost low, this problem cannot be solved directly for each value of the sample $m_{\mathcal{E}}$. 


\subsubsection{Introduction of a reduced basis}

In order to reduce the size of the problem to be solved, one begins by introducing a reduced modal basis by keeping only the $n$ eigenmodes such that:

$$
\bar{e}_{\mathcal{E}}\left(\bar{\phi}_{i}\right) \geq k_{\%} \bar{e}\left(\bar{\phi}_{i}\right)
$$

where $\bar{e}\left(\bar{\phi}_{i}\right)$ is the total strain energy associated with Eigenmode $\underline{\phi}_{i}$. Typically, one chooses $k_{\%}=0.3$.

In order to improve the results with this reduced basis, one introduces some additional static modes $\underline{\psi}_{i}$ defined by:

$$
\overline{\mathbf{K}} \underline{\psi}_{i}=\Delta \mathbf{K}_{\mathcal{E}} \bar{\phi}_{i}
$$

After eliminating collinear modes and normalizing the static modes with respect to the mass matrix, orthogonality relations for the $m \leq n$ calculated static modes are derived:

$$
\forall i, j \quad \underline{\psi}_{j}^{T} \mathbf{M} \underline{\bar{\phi}}_{i}=0 \quad \text { and } \quad \underline{\psi}_{j}^{T} \mathbf{K} \bar{\phi}_{i}=0
$$

Then, the solution of the initial problem is sought as follows:

$$
\underline{\Phi}_{i}=\boldsymbol{\Phi} \underline{x}+\boldsymbol{\Psi} \underline{y}=(\boldsymbol{\Phi} \mid \boldsymbol{\Psi})\left(\frac{\underline{x}}{\underline{y}}\right)
$$

where $\boldsymbol{\Phi}=\left(\underline{\bar{\phi}}_{1} \ldots \underline{\bar{\phi}}_{n}\right)$ and $\boldsymbol{\Psi}=\left(\underline{\psi}_{1} \ldots \underline{\psi}_{m}\right)$.

By premultiplying the previous equality by $(\boldsymbol{\Phi} \mid \boldsymbol{\Psi})^{T}$, one finally gets:

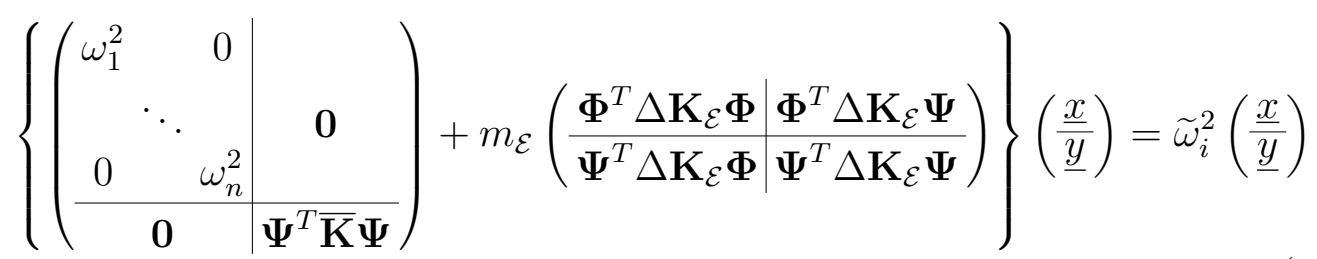

\subsubsection{Interpolation}

In addition to the reduction of computation time due to the size of this reduced system, the values $\widetilde{\omega}_{i}\left(m_{\mathcal{E}}\right)$ and $\widetilde{\phi}_{i}\left(m_{\mathcal{E}}\right)$ are estimated by interpolation.

Typically, one uses a quadratic interpolation defined over each interval $\left[-\bar{m}_{\mathcal{E}}^{-} ; 0\right]$ or $\left[0 ; \bar{m}_{\mathcal{E}}^{+}\right]$depending on the sign of the sample $m_{\mathcal{E}}$. The approximate values of 
$\widetilde{\omega}_{i}\left(m_{\mathcal{E}}\right)$ and $\widetilde{\phi}_{i}\left(m_{\mathcal{E}}\right)$ are derived from the exact solutions of the reduced system for the following values of the sample $m_{\mathcal{E}}$, depending on its sign:

$$
\left\{-\bar{m}_{\mathcal{E}}^{-} ;-\frac{2 \bar{m}_{\mathcal{E}}^{-}}{3} ;-\frac{\bar{m}_{\mathcal{E}}^{-}}{3}\right\} \quad \text { and } \quad\left\{\frac{\bar{m}_{\mathcal{E}}^{+}}{3} ; \frac{2 \bar{m}_{\mathcal{E}}^{+}}{3} ; \bar{m}_{\mathcal{E}}^{+}\right\}
$$

From this interpolation, the strain energies are estimated using a fifth-order approximation:

$$
\widetilde{e}=\frac{1}{2} \widetilde{\phi}_{i}^{T}\left(\overline{\mathbf{K}}+m_{\mathcal{E}} \Delta \mathbf{K}_{\mathcal{E}}\right) \underline{\Phi}_{i}=\sum_{E \in \Omega} \widetilde{e}_{\mathrm{E}}\left(\widetilde{\phi}_{i}\left(m_{\mathcal{E}}\right)\right)
$$

Finally, it is possible to combine the other basic LOKs simply by adding their contributions to the model perturbed by the large value of the basic LOK on $\mathcal{E}$. For instance, we derive the following bounds for the eigenfrequencies:

$$
\begin{aligned}
& {\left[\Delta \omega_{i \text { mod }}^{2-}\right](\theta)=2 \sum_{E \neq \mathcal{E}} m_{\mathrm{E}}^{-}(\theta) \widetilde{e}_{\mathrm{E}}\left(\widetilde{\phi}_{i}\left(m_{\mathcal{E}}(\theta)\right)\right)-\left(\widetilde{\omega}_{i}\left(m_{\mathcal{E}}(\theta)\right)^{2}-\bar{\omega}_{i}^{2}\right)} \\
& {\left[\Delta \omega_{i \text { mod }}^{2+}\right](\theta)=2 \sum_{E \neq \mathcal{E}} m_{\mathrm{E}}^{+}(\theta) \widetilde{e}_{\mathrm{E}}\left(\widetilde{\phi}_{i}\left(m_{\mathcal{E}}(\theta)\right)\right)+\widetilde{\omega}_{i}\left(m_{\mathcal{E}}(\theta)\right)^{2}-\bar{\omega}_{i}^{2}}
\end{aligned}
$$

This method will be applied to an academic example in Section 5 and to an actual industrial structure in Section 6.

\section{Identification and reduction of the basic LOKs}

\subsection{Comparison with an experimental reference}

Let us consider a structural model with LOKs giving for the quantity of interest $\alpha$ the two bounds:

$$
\left[\Delta \alpha_{\text {mod }}^{+}\right](\theta),\left[\Delta \alpha_{\text {mod }}^{-}\right](\theta)
$$

The quantification of the quality of a model with respect to an experimental reference using our theory involves the determination of the basic LOKs which best characterize the experimental variabilities. Regarding the family of actual structures, one can determine two values $\Delta \alpha_{\exp }^{-}$and $\Delta \alpha_{\exp }^{+}$which, for a given probability $P$, contain $P \%$ of the values of the quantity of interest $\Delta \alpha_{\exp }$ related to the actual structures. 
Then, using the concept of interval probability, one can compare the experimental data with the values given by the LOK model by saying that the basic LOKs must be such that:

$$
P\left(\Delta \alpha_{\text {exp }} \in I_{\Delta \alpha_{\text {mod }}}(L)\right) \geq P_{\alpha}(L) \forall L
$$

Through this relation, we express that the LOK model should be determined conservatively, i.e. that the interval probability, which is a lower bound of the actual probability of $\Delta \alpha_{\text {mod }}$ being within the standard interval $I_{\Delta \alpha_{\text {mod }}}\left(L_{\mathrm{P}}\right)$, should be a lower bound of the probability of the experimental quantity of interest $\Delta \alpha_{\text {exp }}$ being within $I_{\Delta \alpha_{\text {mod }}}\left(L_{\mathrm{P}}\right)$.

This necessary condition is the same as saying, in terms of standard intervals, that the relation $\Delta \alpha_{\text {exp }}^{-}(P) \leq \Delta \alpha_{\text {mod }}^{-}(P)$ and $\Delta \alpha_{\text {exp }}^{+}(P) \leq \Delta \alpha_{\text {mod }}^{+}(P)$ must be verified for any given probability $P$. This interpretation is, in fact, a generalization of the $99 \%$-values mentioned in [15].

\subsection{Reduction of the basic LOKs}

\subsubsection{Main principle}

The main idea behind this determination is that the greater the amount of experimental information available, the more likely one is to reduce the basic LOKs. This principle requires an initial description, which may be coarse but must necessarily be overestimated, of the basic LOKs for each substructure. This can be obtained through a priori knowledge or through experiments relevant to the structure being studied. In the end, one has a set of initial basic LOKs $\left(m_{\mathrm{E}}^{+0}(\theta), m_{\mathrm{E}}^{-0}(\theta)\right)_{E \in \Omega}$ (associated with probability laws) which are such that all the constraints (43) are verified for the experimental data available, and which can be used to describe the basic LOKs for each substructure.

In our present approach, which is conservative, the reduction process consists in using this additional relevant experimental information to reduce the level of LOK one substructure at a time. Let us consider a particular substructure $E^{*}$. The problem is to determine a basic LOK $\left(m_{\mathrm{E}^{*}}^{-}(\theta), m_{\mathrm{E}^{*}}^{+}(\theta)\right)$ smaller than the initial $\mathrm{LOK}\left(m_{\mathrm{E}^{*}}^{-0}(\theta), m_{\mathrm{E}^{*}}^{+0}(\theta)\right)$, which is equivalent, in terms of interval probabilities, to the following inequality:

$$
P_{E^{*}}^{0}(L) \leq P_{E^{*}}(L) \quad \forall L
$$

This reduction is carried out under Constraint (43) associated with the experimental information chosen, which, in terms of the effective LOK on the quantity of interest $\alpha$, can be written as:

$$
\Delta \alpha_{\exp }^{-}(P) \leq \Delta \alpha_{\text {mod }}^{-}(P) \text { and } \Delta \alpha_{\exp }^{+}(P) \leq \Delta \alpha_{\text {mod }}^{+}(P)
$$




\subsubsection{Worst-case estimates of $\Delta \alpha_{\mathrm{mod}}^{+}$and $\Delta \alpha_{\mathrm{mod}}^{-}$}

Since the reduction is carried out one substructure at a time, the verification of Constraint (43) is not sufficient to guarantee realistic results in all situations. Indeed, let us recall that the effective LOK derives from the interval probability $P_{\alpha}(L)$ which characterizes the distribution of the bounds $\left[\Delta \alpha_{\bmod }^{+}(\theta)\right]$ and $\left[\Delta \alpha_{\bmod }^{-}(\theta)\right]$ of the quantity of interest $\Delta \alpha_{\text {mod }}$ relative to the model. As these bounds contain distinct contributions from all the substructures, for a given set of samples $\left(m_{\mathrm{E}}^{-}, m_{\mathrm{E}}^{+}\right)_{E \in \Omega}$, one has:

$$
\Delta \alpha_{\mathrm{mod}}=\widehat{\alpha}\left(\overline{\mathbb{A}}\left(\mathbf{K}_{\mathrm{E}} ; E \in \Omega\right)\right)-\bar{\alpha}=\Delta \alpha_{E^{*}}+\sum_{E \neq E^{*}} \Delta \alpha_{E}
$$

In our reduction process, we build worst-case estimates of $\left[\Delta \alpha_{\text {mod }}^{+}(\theta)\right]$ and $\left[\Delta \alpha_{\text {mod }}^{-}(\theta)\right]$, denoted $\left[\Delta \alpha_{\text {mod }}^{+ \text {worst }}(\theta)\right]$ and $\left[\Delta \alpha_{\text {mod }}^{- \text {worst }}(\theta)\right]$, which verify:

$$
\Delta \alpha_{\bmod }^{- \text {worst }}(P) \leq \Delta \alpha_{\text {exp }}^{-}(P) \text { and } \Delta \alpha_{\text {mod }}^{+ \text {worst }}(P) \leq \Delta \alpha_{\exp }^{+}(P)
$$

These worst-case values must lie within the experimental bounds.

Precisely, we write:

$$
\begin{aligned}
& \Delta \alpha_{\text {mod }}^{- \text {worst }}=\Delta \alpha_{E^{*}}^{-}+\Delta \alpha_{\mathfrak{C}_{E^{*}}^{- \text {worst }}}^{-} \\
& \Delta \alpha_{\text {mod }}^{+ \text {worst }}=\Delta \alpha_{E^{*}}^{+}+\Delta \alpha_{\complement_{E^{*}}}^{+ \text {worst }}
\end{aligned}
$$

where

$$
\begin{aligned}
-\Delta \alpha_{\mathrm{C}_{E^{*}}}^{- \text {worst }} & =\max _{-m_{\mathrm{E}}^{-} \overline{\mathbf{K}}_{\mathrm{E} \leq \mathbf{K}_{\mathrm{E}}-\overline{\mathbf{K}}_{\mathrm{E}} \leq m_{\mathrm{E}}^{+} \overline{\mathbf{K}}_{\mathrm{E}}}} \widehat{\alpha}\left(\overline{\mathbb{A}}\left(\mathbf{K}_{\mathrm{E}} ; E \in \Omega\right)\right)-\bar{\alpha} \\
\Delta \alpha_{\mathrm{C}_{E^{*}}^{+ \text {worst }}} & =\min _{-m_{\mathrm{E}}^{-} \overline{\mathbf{K}}_{\mathrm{E}} \leq \mathbf{K}_{\mathrm{E}}-\overline{\mathbf{K}}_{\mathrm{E}} \leq m_{\mathrm{E}}^{+} \overline{\mathbf{K}}_{\mathrm{E}}} \widehat{\alpha}\left(\overline{\mathbb{A}}\left(\mathbf{K}_{\mathrm{E}} ; E \in \Omega\right)\right)-\bar{\alpha}
\end{aligned}
$$

For example, with regard to the effective LOK on the square of an eigenfrequency $\omega_{i}^{2}$, one has to deal with:

$$
\begin{aligned}
& {\left[\Delta \alpha_{\mathrm{E}}^{- \text {worst }}(\theta)\right]=-2 m_{\mathrm{E}}^{+}(\theta) \bar{e}_{\mathrm{E}}\left(\bar{\phi}_{i}\right)} \\
& {\left[\Delta \alpha_{\mathrm{E}}^{+ \text {worst }}(\theta)\right]=-2 m_{\mathrm{E}}^{-}(\theta) \bar{e}_{\mathrm{E}}\left(\underline{\bar{\phi}}_{i}\right)}
\end{aligned}
$$

\subsubsection{Representativeness of the experimental data}

Until now, we assumed implicitly that the experimental data we used in the reduction process gave a good description of the basic LOKs on the different substructures. In reality, the information given by experimental data is generally only partial. 
Therefore, we allow our worst-case analysis to be further enriched by introducing a coefficient $\left.\left.\rho_{E^{*}} \in\right] 0 ; 1\right]$ which enables one to quantify the extent to which the selected experimental information is representative of the behavior of the substructure: this quantity, which we call the coefficient of representativeness, is maximum when the experimental data give a perfect account of the global mechanics of the substructure, including the main sources of error. To illustrate this point, let us consider the example of a traction test: in the case of an isotropic substructure model, the experimental data associated with this test are relevant because they give a trustworthy vision of the LOK associated with the substructure. On the contrary, in the case of an orthotropic model, the same data, by giving information in the direction of the traction test alone, provide only a very partial vision. Therefore, the corresponding bounds are defined as follows:

$$
\begin{aligned}
\Delta \alpha_{\text {mod }}^{- \text {worst }} & =\rho_{E^{*}} \Delta \alpha_{E^{*}}^{-}+\Delta \alpha_{\mathrm{C}_{E^{*}}^{- \text {worst }}}^{-} \\
\Delta \alpha_{\text {mod }}^{+ \text {worst }} & =\rho_{E^{*}} \Delta \alpha_{E^{*}}^{+}+\Delta \alpha_{\mathrm{C}_{E^{*}}}^{+ \text {worst }}
\end{aligned}
$$

The value of the coefficient $\rho_{E^{*}}$, which belongs to $\left.] 0 ; 1\right]$, is closely related to the type of test being considered. 1 corresponds to a perfectly representative test. Let us observe that a precise value is not necessary and that specialists should be able to propose a reasonable coefficient for any given specific case. In several cases in which the sources of error are well-established, the coefficient of representativeness can be obtained from calculations.

The worst evaluations of $\Delta \alpha_{\text {mod }}$ defined by (48) should verify Inequalities (47). These and Inequalities (45) are the constraints related to the inverse problem defining the new set of LOKs for Substructure $E^{*}$. It is important to understand that if the test has not been chosen well, the reduction process does not lead to any reduction: its solution is the initial set of LOKs for Substructure $E^{*}$.

\section{First example}

\subsection{Definition of the structure}

Let us consider a family of similar, but not identical, plane trusses, each consisting of six pin-jointed bars as shown in Figure 1. 


\subsubsection{Data of the theoretical model}

The theoretical model is defined as a FE model with deterministic characteristics. The bars are assumed to be solicited only in traction-compression and the connections between the structure and the base are assumed to be perfectly rigid. The material characteristics of the theoretical model are given in Table 1 .

\subsubsection{Experimental data}

In order to derive the experimental data which enable the reduction of the basic LOKs, we used the eigenfrequencies and eigenmodes of a family of similar trusses. In order to simulate these "experimental" trusses, we introduced stiffness dispersions into the material characteristics of the theoretical model as defined in Table 1. One should note that Material "X" is not known very well and that the associated stiffness dispersion follows a uniform distribution. Then, we calculated the eigenfrequencies and eigenmodes of each of these simulated trusses and determined their distribution laws (e.g. Figure 2), which we took as the experimental quantities of interest.

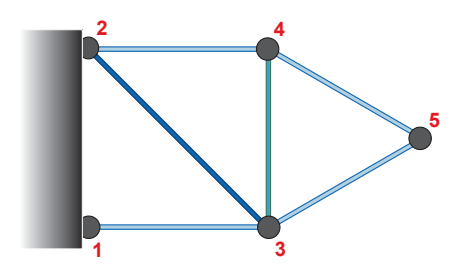

Fig. 1. The plane truss being studied

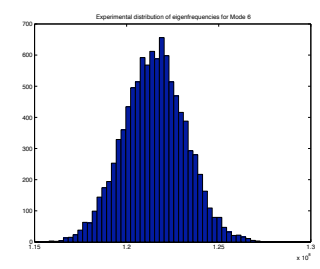

Fig. 2. Experimental distribution of $\omega_{\text {exp }}^{2}$ for Mode 6

Table 1

Material characteristics of the deterministic model of the truss and of the numerically simulated structures, where Group g1 consists of bars 1-3, 3-5, 4-5 and 2-4, bar 2-3 is Group g2 and bar 3-4 is Group g3

\begin{tabular}{|c||c|c|c||c|c|c|}
\hline Gr. & $\begin{array}{c}\text { Mate- } \\
\text {-rial }\end{array}$ & $\begin{array}{c}\text { Young's } \\
\text { modulus }\end{array}$ & $\begin{array}{c}\text { Mass } \\
\text { density }\end{array}$ & $\begin{array}{c}\text { Law } \\
\text { chosen }\end{array}$ & $\begin{array}{c}\text { Relative } \\
\text { range }\end{array}$ & $\begin{array}{c}\text { Relative statistical moments } \\
(\mu \text { : mean } / \sigma \text { : std. dev. })\end{array}$ \\
\hline$g 1$ & alum. & $\bar{E}_{g 1}=72 \mathrm{GPa}$ & $2,700 \mathrm{~kg} / \mathrm{m}^{3}$ & normal & {$[-0.05 ; 0.05]$} & $\mu=0.00 / \sigma=0.019$ \\
$g 2$ & steel & $\bar{E}_{g 2}=210 \mathrm{GPa}$ & $7,800 \mathrm{~kg} / \mathrm{m}^{3}$ & normal & {$[-0.20 ; 0.70]$} & $\mu=0.25 / \sigma=0.175$ \\
$g 3$ & "X" & $\bar{E}_{g 3}=10 \mathrm{GPa}$ & $1,500 \mathrm{~kg} / \mathrm{m}^{3}$ & uniform & {$[-0.15 ; 0.05]$} & $\mu=-0.05 / \sigma=0.039$ \\
\hline
\end{tabular}




\subsection{Reduction of the basic LOKs}

The reduction process was initiated by prescribing a priori on each substructure initial LOK values which constitute appropriate overestimated levels; these levels are indicated in Table 2. We also assumed that the probability laws of the basic LOKs were normal for the aluminum and steel bars and uniform for the bar made of Material "X". The only experimental information retained was the values $\Delta \omega_{i \exp }^{2+}(0.99)$ and $\Delta \omega_{i \exp }^{2-}(0.99)$ which bounded $99 \%$ of the experimental values of the eigenfrequencies actually measured; in particular, we did not concern ourselves with the distribution of these values between these bounds of the standard $99 \%$-probability interval $I_{\Delta \omega_{i \bmod }^{2}}$. If one were to seek a richer description, one could also use the 70\%-probability values of these experimental quantities, which would provide an estimate of the standard deviation.

Table 2

Initial LOK model $\left(m_{\mathrm{E}}^{-0}, m_{\mathrm{E}}^{+0}\right)$

\begin{tabular}{|c|c|c|c|}
\hline Groups & $\begin{array}{c}\text { Law } \\
\text { chosen }\end{array}$ & $\begin{array}{c}\text { Range } \\
{\left[-\bar{m}_{\mathrm{E}}^{-0} ; \bar{m}_{\mathrm{E}}^{+0}\right]}\end{array}$ & $\begin{array}{c}\text { Relative statistical moments } \\
(\mu \text { : mean / } \sigma \text { : std. dev. })\end{array}$ \\
\hline$g 1=\{b 13, b 24, b 35, b 45\}$ & normal & {$[-0.25 ; 0.25]$} & $\mu=0.00 / \sigma=0.097$ \\
$g 2=\{b 23\}$ & normal & {$[-0.75 ; 0.75]$} & $\mu=0.00 / \sigma=0.289$ \\
$g 3=\{b 34\}$ & uniform & {$[-0.25 ; 0.25]$} & $\mu=0.00 / \sigma=0.097$ \\
\hline
\end{tabular}

Regarding the second step, it was important to identify the best experimental measurements in order to carry out reductions on the different substructures successfully. An effective method consists in relying upon the fact that the sensitivity of the effective LOKs with respect to the basic LOKs is directly related to the modal strain energies of the deterministic theoretical model, as can be seen in the expressions of Section 3.2. Indeed, the most relevant experimental data in the process of reducing the basic LOKs on Structure $E^{*}$ are those whose modal strain energy is located primarily in $E^{*}$. Since the experimental information we chose concerned eigenfrequencies, we needed to consider the energies $\bar{e}_{\mathrm{E}}\left(\underline{\phi}_{i}\right)$, which are depicted in Figure 3.

The reduction process was carried out by choosing successively Modes 6, 4 and 2 (for Groups $g 1, g 2$ and $g 3$ respectively) as the experimental data and by assuming that these data were representative of the global behavior of the truss (coefficients $\rho_{E}$ equal to 1 ). The final results are given in Table 3.

For this simple case, these results can be compared directly to the stiffness dispersions introduced into the deterministic model in order to simulate the experimental data: $\left[\bar{K}_{g 1}-5 \% ; \bar{K}_{g 1}+5 \%\right],\left[\bar{K}_{g 2}-20 \% ; \bar{K}_{g 2}+70 \%\right]$ and $\left[\bar{K}_{g 3}-15 \% ; \bar{K}_{g 3}+5 \%\right]$. While the reduced basic LOKs for Groups $g 1$ and $g 3$ 


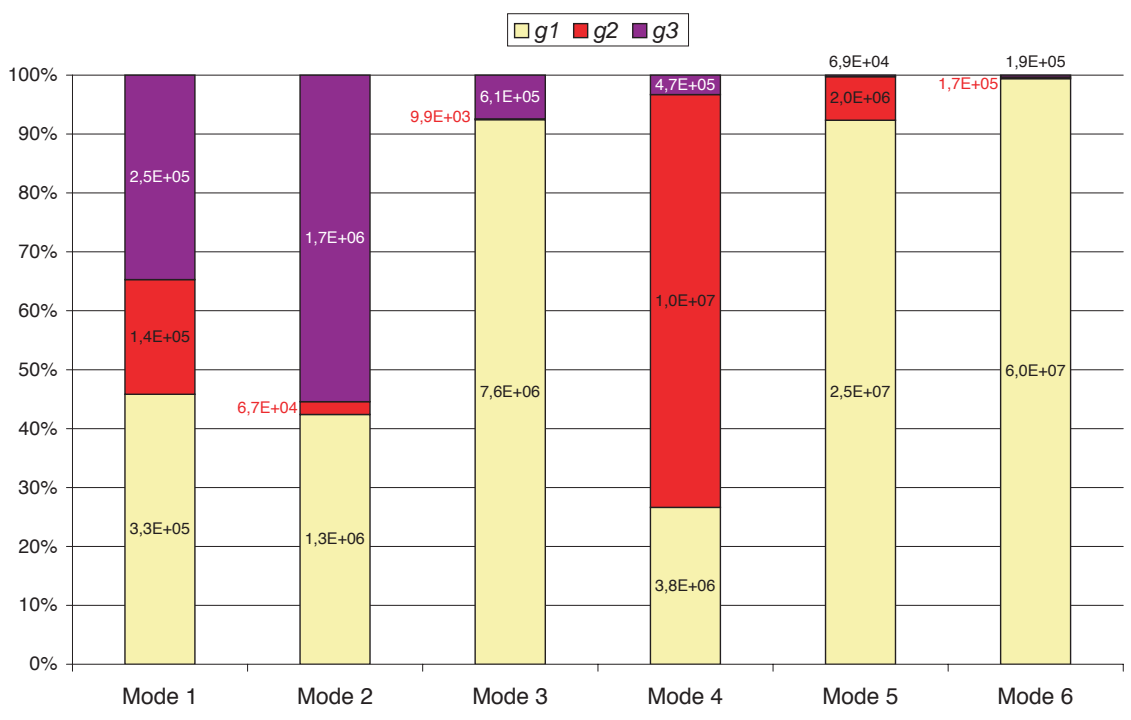

Fig. 3. Modal strain energies for Modes 1 to 6

Table 3

Reduced basic LOKs

\begin{tabular}{|c|c|c|c|c|}
\hline \multirow{2}{*}{ Groups } & Experimental data & \multicolumn{3}{|c|}{ Reduced basic LOKs } \\
\cline { 3 - 5 } & & Law & {$\left[-\bar{m}_{\mathrm{E}}^{-} ; \bar{m}_{\mathrm{E}}^{+}\right]$} & Statistical moments \\
\hline$g 1$ & $\left(\Delta \omega_{6 \exp }^{2+}(0.99), \Delta \omega_{6 \exp }^{2-}(0.99)\right)$ & normal & {$[-0.032 ; 0.034]$} & $\mu=0.001 / \sigma=0.013$ \\
$g 2$ & $\left(\Delta \omega_{4 \exp }^{2+}(0.99), \Delta \omega_{4 \exp }^{2-}(0.99)\right)$ & normal & {$[-0.148 ; 0.581]$} & $\mu=0.217 / \sigma=0.142$ \\
$g 3$ & $\left(\Delta \omega_{2 \exp }^{2+}(0.99), \Delta \omega_{2 \exp }^{2-}(0.99)\right)$ & uniform & {$[-0.165 ; 0.075]$} & $\mu=-0.045 / \sigma=0.035$ \\
\hline
\end{tabular}

are quite good, the value obtained for Group $g 2$ is not very accurate compared to the corresponding experimental dispersion. The fact that this value is large suggests that one should start the reduction process from scratch, this time using the calculation method which takes large LOK values into account instead of a linearization technique.

Modes 1 to 4 were used in the reduced basis involved in the specific process described in Section 3.3. Once again, the reduction process was carried out by choosing successively Modes 6, 4 and 2 (for Groups $g 1, g 2$ and $g 3$ respectively) as the experimental data and by assuming that these data were representative of the global behavior of the truss (coefficients $\rho_{E}$ equal to 1 ). The corresponding results are shown in Table 4 . This time, one can observe the good agreement of the values obtained for Group $g 2$. 
Table 4

Reduced basic LOKs taking into account large values of the LOK on Group $g 2$

\begin{tabular}{|c|c|c|c|c|}
\hline \multirow{2}{*}{ Groups } & Experimental & \multicolumn{3}{|c|}{ Reduced basic LOKs } \\
\cline { 3 - 5 } & data & Law & {$\left[-\bar{m}_{\mathrm{E}}^{-} ; \bar{m}_{\mathrm{E}}^{+}\right]$} & Statistical moments \\
\hline$g 1$ & $\left(\Delta \omega_{6 \exp }^{2+}(0.99), \Delta \omega_{6 \exp }^{2-}(0.99)\right)$ & normal & {$[-0.032 ; 0.034]$} & $\mu=0.001 / \sigma=0.013$ \\
$g 2$ & $\left(\Delta \omega_{4 \exp }^{2+}(0.99), \Delta \omega_{4 \exp }^{2-}(0.99)\right)$ & normal & {$[-0.195 ; 0.703]$} & $\mu=0.254 / \sigma=0.174$ \\
$g 3$ & $\left(\Delta \omega_{2 \exp }^{2+}(0.99), \Delta \omega_{2 \exp }^{2-}\right)(0.99)$ & uniform & {$[-0.155 ; 0.075]$} & $\mu=-0.040 / \sigma=0.033$ \\
\hline
\end{tabular}

6 Study of an industrial case

Now, let us examine the application of our method to an actual industrial structure: the Sylda5 satellite support capable of carrying two satellites simultaneously, developed by EADS Group (Figure 4).

\subsection{Description of the structure}

\subsubsection{Experimental data}

Free-vibration measurements with 260 sensors were carried out by IABG on behalf of DASA/DORNIER under contract with CNES.

\subsubsection{Data for the theoretical model}

The model proposed by EADS represents both the support itself and a cylindrical payload which simulates the presence of a satellite resting on the support. This model consists of 38 substructures made of various materials, including orthotropic sandwich materials, aluminum and steel. Since initial measurements had shown that it was absolutely essential to take into account the deformation of the ground under the support, this was modeled very simply using 3 torsional springs, one translational spring and a rigid-body constraint for all the nodes at the junction between the ground and the support. The final model consisted of 27,648 DOFs and 9,728 elements.

\subsection{Determination of the basic LOKs}

First, based on the first 12 experimental modes, the model was calibrated using the method described in [2]. The problem was then to determine the remaining LOKs. In order to do that, the structure was divided into 4 main groups of substructures, as described in Figure 5: 


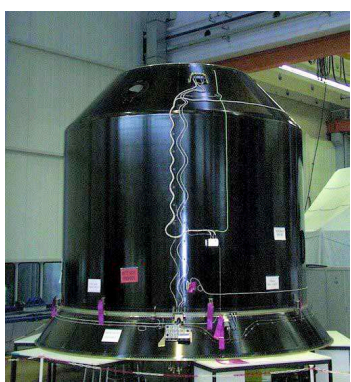

Fig. 4. Photograph of the Sylda5 support

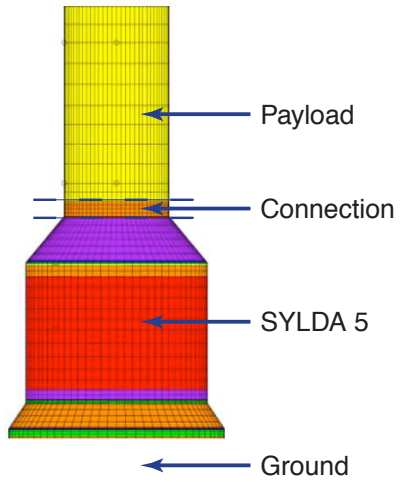

Fig. 5. The associated Sylda5 model

- Group $g 1$ associated with the cylindrical payload;

- Group g2 containing the composite connection between the cylinder and the Sylda5 support;

- Group g3 corresponding to the Sylda5 support itself;

- Group g4 associated with the ground model.

The reduction process was initiated by setting a priori the initial LOK levels $\left(\bar{m}_{\mathrm{E}}^{-0}, \bar{m}_{\mathrm{E}}^{+0}\right)$ (and their corresponding laws) as shown in Table 5. The experimental information consisted of the eigenmodes and eigenfrequencies measured on the actual structure, which were considered as the extreme values that would have been obtained if several similar structures had been tested.

Table 5

Initial LOK model

\begin{tabular}{|c|c|c|c|}
\hline Groups & $\begin{array}{c}\text { Law } \\
\text { being sought }\end{array}$ & $\begin{array}{c}\text { Range } \\
\left(\bar{m}_{\mathrm{E}}^{-0}, \bar{m}_{\mathrm{E}}^{+0}\right)\end{array}$ & $\begin{array}{c}\text { Relative statistical moments } \\
(\mu \text { : mean } / \sigma \text { : std. dev. })\end{array}$ \\
\hline $\mathrm{E}=g 1$ & normal & $(-0.25,0.25)$ & $(\mu=0.00 / \sigma=0.097)$ \\
$\mathrm{E}=g 2$ & uniform & $(-0.25,0.25)$ & $(\mu=0.00 / \sigma=0.097)$ \\
$\mathrm{E}=g 3$ & normal & $(-0.25,0.25)$ & $(\mu=0.00 / \sigma=0.097)$ \\
$\mathrm{E}=g 4$ & uniform & $(-0.75,0.75)$ & $(\mu=0.00 / \sigma=0.289)$ \\
\hline
\end{tabular}

Table 6 shows the order in which the reduction was carried out, the data which were used and the results (with $\rho_{E}=1$ ) of the process.

These results confirmed the good quality of the calibrated model of the support (g3) and of the model of the connector (both within a few \%), but pointed out the oversimplifications in the ground model resulting in a large LOK, which suggested the use of the specific process described in Section 3.3.

The whole reduction process was started again from scratch, using a reduced basis consisting of the first eight modes of the structure and the same experimental data as before. The corresponding results are given in Table 7 . 
Table 6

Reduced basic LOKs

\begin{tabular}{|c|c|c|c|c|}
\hline \multirow{2}{*}{ Groups } & Experimental & \multicolumn{3}{|c|}{ Reduced basic LOKs } \\
\cline { 3 - 5 } & data & Law & {$\left[-\bar{m}_{\mathrm{E}}^{-} ; \bar{m}_{\mathrm{E}}^{+}\right]$} & Statistical moments \\
\hline$g 3$ & $\left(\Delta \omega_{4 \exp }^{2+}(0.99), \Delta \omega_{4 \exp }^{2-}(0.99)\right)$ & normal & {$[-0.016 ; 0.000]$} & $\mu=-0.008 / \sigma=0.003$ \\
$g 1$ & $\left(\Delta \omega_{8 \exp }^{2+}(0.99), \Delta \omega_{8 \exp }^{2-}(0.99)\right)$ & normal & {$[0.000 ; 0.144]$} & $\mu=0.072 / \sigma=0.028$ \\
$g 4$ & $\left(\Delta \omega_{6 \exp }^{2+}(0.99), \Delta \omega_{6 \exp }^{2-}(0.99)\right)$ & uniform & {$[0.000 ; 0.435]$} & $\mu=0.218 / \sigma=0.126$ \\
$g 2$ & $\left(\Delta \omega_{3 \exp }^{2+}(0.99), \Delta \omega_{3 \exp }^{2-}(0.99)\right)$ & uniform & {$[-0.060 ; 0.000]$} & $\mu=-0.030 / \sigma=0.012$ \\
\hline
\end{tabular}

Table 7

Reduced basic LOKs taking into account large values of the LOK for the ground (Group g4)

\begin{tabular}{|c|c|c|c|c|}
\hline \multirow{2}{*}{ Groups } & Experimental & \multicolumn{3}{|c|}{ Reduced basic LOKs } \\
\cline { 3 - 5 } & data & Law & {$\left[-\bar{m}_{\mathrm{E}}^{-} ; \bar{m}_{\mathrm{E}}^{+}\right]$} & Statistical moments \\
\hline$g 3$ & $\left(\Delta \omega_{4 \exp }^{2+}(0.99), \Delta \omega_{4 \exp }^{2-}(0.99)\right)$ & normal & {$[-0.016 ; 0.000]$} & $\mu=-0.008 / \sigma=0.003$ \\
$g 1$ & $\left(\Delta \omega_{8 \exp }^{2+}(0.99), \Delta \omega_{8 \exp }^{2-}(0.99)\right)$ & normal & {$[0.000 ; 0.144]$} & $\mu=0.072 / \sigma=0.028$ \\
$g 4$ & $\left(\Delta \omega_{6 \exp }^{2+}(0.99), \Delta \omega_{6 \exp }^{2-}(0.99)\right)$ & uniform & {$[0.000 ; 0.521]$} & $\mu=0.261 / \sigma=0.150$ \\
$g 2$ & $\left(\Delta \omega_{3 \exp }^{2+}(0.99), \Delta \omega_{3 \exp }^{2-}(0.99)\right)$ & uniform & {$[-0.060 ; 0.000]$} & $\mu=-0.030 / \sigma=0.012$ \\
\hline
\end{tabular}

Except for the LOK associated with the ground, the basic LOKs of the other groups were unchanged. In order to evaluate the quality of the results of the reduction process, one can calculate the effective LOK for Mode 1, which was not used, and compare this with the corresponding experimental values from Table 8. One can observe that the constraints for Mode 1 are properly verified, which shows that the results obtained with the other modes were consistent. Table 8

Comparison of 99\%-values for Mode 1

\begin{tabular}{|c||c|c|c|c|c|}
\hline$i$ & $\bar{\omega}_{i}^{2}+\Delta \omega_{i \bmod }^{2-}$ & $\bar{\omega}_{i}^{2}+\Delta \omega_{i \exp }^{2-}$ & $\bar{\omega}_{i}^{2}$ & $\bar{\omega}_{i}^{2}+\Delta \omega_{i \exp }^{2+}$ & $\bar{\omega}_{i}^{2}+\Delta \omega_{i \bmod }^{2+}$ \\
\hline 1 & $1.0110^{3}$ & $1.0210^{3}$ & $1.0210^{3}$ & $1.0510^{3}$ & $1.0610^{3}$ \\
\hline
\end{tabular}

\section{Conclusion}

We developed a new approach to structural modeling using LOKs which consists in inserting validity indicators into the model in the form of basic LOKs, then predicting the "envelope" of the responses of the family of actual structures being considered. We presented examples which illustrate how this approach works and what its capabilities are. The extension to sources of error other than stiffness errors is in progress. Such a modeling scheme is suitable for robust design [26]. 


\section{References}

[1] J. E. Mottershead, M. I. Friswell, Model updating in structural dynamics: a survey, Journal of Sound and Vibration 167 (2) (1993) 347-375.

[2] P. Ladevèze, A. Chouaki, Application of a posteriori error estimation for structural model updating, Inverse Problems 15 (1999) 49-58.

[3] A. Deraemaeker, P. Ladevèze, T. Romeuf, Model validation in the presence of uncertain experimental data, Engineering Computations 21 (8) (2004) 808-833.

[4] P. Ladevèze, G. Puel, A. Deraemaeker, T. Romeuf, Validation of structural dynamics model with uncertainties, Computer Methods in Applied Mechanics and Engineering(to appear 2005).

[5] F. M. Hemez, S. W. Doebling, M. C. Anderson, A brief tutorial on verification and validation, in: Proceedings of the 22th International Modal Analysis Conference (IMAC-XXII), Dearborn, Michigan, 2004.

[6] G. I. Schuëller, Computational stochastic mechanics - recent advances, Computers and Structures 79 (2001) 2225-2234.

[7] R. G. Ghanem, P. D. Spanos, Stochastic Finite Elements: a Spectral Approach, Springer, 1991.

[8] R. Ghanem, Ingredients for a general purpose stochastic finite elements implementation, Computer Methods in Applied Mechanics and Engineering 168 (1999) 19-34.

[9] H. G. Matthies, A. Keese, Galerkin methods for linear and nonlinear elliptic stochastic partial differential equations, Computer Methods in Applied Mechanics and Engineering 194 (2005) 1295-1331.

[10] I. Babuska, R. Tempone, G. E. Zouraris, Solving elliptic boundary value problems with uncertain coefficients by the finite element method: The stochastic formulation, Computer Methods in Applied Mechanics and Engineering 194 (2005) 1251-1294.

[11] J. Argyris, M. Papadrakakis, G. Stefanou, Stochastic finite element analysis of shells, Computer Methods in Applied Mechanics and Engineering 191 (2002) 4781-4804.

[12] Y. Ben-Haim, Information-Gap Decision Theory, Academic Press, London, 2001.

[13] G. Klir, Generalized information theory, Fuzzy Sets and Systems 40 (1991) $127-142$.

[14] P. Walley, Statistical Reasoning with Imprecise Probabilities, Chapman and Hall, 1990. 
[15] D. Barthe, A. Deraemaeker, P. Ladevèze, G. Puel, On a theory of the quantification of the lack of knowledge in structural computation, in: Proceedings of the 21st International Modal Analysis Conference (IMAC-XXI), Kissimmee, Florida, 2003.

[16] P. Ladevèze, G. Puel, T. Romeuf, On a strategy of reduction of the lack of knowledge (LOK) of a structural dynamics model, in: Proceedings of IMAC XXII, Dearborn, Michigan, 2004.

[17] P. Ladevèze, G. Puel, T. Romeuf, On a strategy of reduction of the lack of knowledge (LOK) in model validation, in: Proceedings of the Fourth International Conference on Sensitivity Analysis of Modeling Output, Santa Fe, New Mexico, 2004.

[18] G. Puel, P. Ladevèze, T. Romeuf, How to reduce the lack of knowledge of an industrial model in structural dynamics, in: Proceedings of the 23rd International Modal Analysis Conference (IMAC-XXIII), Orlando, Florida, 2005 .

[19] R. M. Moore, Interval Analysis, Prentice-Hall, 1966.

[20] R. Moore, Methods and Applications of Interval Analysis, Studies in Applied Mathematics (SIAM), 1979.

[21] P. Ladevèze, The theory of the lack of knowledge in structural dynamics: Development and first examples, Technical Note LY22 139 423, EADS Launch Vehicles, in French (May 2003).

[22] R. Nelson, Simplified calculations of eigenvector derivatives, AIAA Journal 14 (1976) 1201-1205.

[23] J. Shaw, S. Jayasuria, Modal sensitivities for repeated eigenvalues and eigenvalues derivatives, AIAA Journal 30 (3) (1992) 850-852.

[24] D. Zhang, F. Wei, Computations of eigenvector derivatives with repeated eigenvalues using a complete modal space, AIAA Journal 33 (9) (1995) 17491753 .

[25] E. Balmès, Efficient sensitivity analysis based on finite element model reduction, in: Proceedings of the 16th International Modal Analysis Conference (IMACXVI), Santa Barbara, California, 1998, pp. 1118-1124.

[26] I. Doltsinis, Z. Kang, Robust design of structures using optimization methods, Computer Methods in Applied Mechanics and Engineering 193 (23-26) (2004) 2221-2237. 